\title{
Improving the energy efficiency of lighting systems by the use of solar radiation
}

\author{
Stawomir Sowa ${ }^{1, *}$ \\ ${ }^{1}$ Institute of Heat Engineering, Faculty of Power and Aeronautical Warsaw University of Technology, Poland
}

\begin{abstract}
The paper presents two ways of using the solar radiation to limit consumption of the electric power for lighting, i.e., increasing the energy efficiency of lighting systems. The solar radiation energy is a generally available type of renewable energy that to a large extent may influence a reduction in consumption of the electric power for lighting. Facilities designers and architects aim at ensuring the highest possible availability of natural lighting in rooms. Usually, lighting systems are not provided with an appropriate control system using the solar radiation. A lack of means for a correct control of the artificial lighting intensity is a factor hindering an improvement of the energy efficiency of the lighting systems. Implementation of the correct control system is possible when distribution of the lighting intensity in a room is known. The studies conducted in this area are a valuable source of information which can be used to develop control algorithms or determine optimum locations and parameters for installation of lighting fixtures. The second way for improving the energy efficiency of the lighting systems is a use of the solar radiation energy converted to the electric power to supply lighting systems.
\end{abstract}

\section{Solar radiation potential}

The solar radiation reaching the Earth surface is composed mainly from the visible light and near-infrared wavelengths. In residential and public utility buildings light plays an important role, significantly influencing a comfort of room use, occupants mood, and quality and safety of performed activities. The magnitude of light intensity reaching a room is also of importance for a type and power of installed lighting fixtures. When an appropriate intensity of natural light is ensured in a given room, then, using the solar radiation we can reduce the electric power consumption by switching off or dimming artificial lighting. The amount of the solar radiation reaching a room depends on many factors. A basic obvious factor is a geographical location of a facility, its orientation, and direct environment. Another aspect resulting from laws of physics is a season and a time of a day. Weather conditions also have a significant influence on intensity of light reaching a room. Other conditions influencing the amount of the solar radiation reaching the room are associated with building architecture.

Relevant factors for delivery of the solar radiation are specified in the standard PrEN 15193 [1]. This standard specifies factors which can be used as a basis for classification of rooms with small, medium and large availability of light. The solar radiation is not just the electromagnetic radiation within the visible spectrum, which we use to light rooms. The solar radiation is also power that can be converted in photovoltaic cells into the electric power. In that case, possibilities for using the solar radiation energy are greater because photovoltaic systems are installed in optimal locations enabling maximum use of the available solar energy. This effective use of the solar radiation for lighting rooms and producing the electric power can significantly reduce consumption of the electric power used for lighting. The use of the solar radiation potential to the highest extent possible will depend on an appropriate selection of a system for control of the lighting system and installation of an efficient photovoltaic system. Ways for using the solar radiation on a basis of results of tests and measurements proving that effectively used solar radiation resources can significantly influence an improvement in the energy efficiency of lighting systems are presented in further sections of this paper. It should also be remembered that the solar radiation exceeding lighting needs of rooms is a negative phenomenon. In these cases we must limit the solar radiation access, preventing negative phenomena, i.e., for example, glare or excessive heating of a room. A reduction in an excessive solar radiation reaching a room can be controlled, for example, by using shutters or blinds.

\section{Tests and measurements location}

Tests of the lighting intensity distribution and measurements of the generated electric power were conducted at an educational facility, the School Complex No. 1 in Swarzędz near Poznań. In a period of 18 months, measurements of the lighting intensity distribution were conducted and the energy generated in the photovoltaic system was recorded. The

\footnotetext{
* Corresponding author: $\underline{\text { Slawomir@sowa.poznan.pl }}$
} 
measurements were conducted in lecturing rooms facing different, opposite directions.

\subsection{Studied facilities}

\subsubsection{Building location}

The school building is a detached facility located near Poznań, of the following coordinates: latitude of 52.3975 $\mathrm{N}$ and longitude of $17.0641 \mathrm{E}$. No external obstacles in form of buildings or landscape features that could limit the flow of the solar radiation to the studied rooms were present at the locations where lighting intensity was measured. This advantageous location ensures reliable measurements independent of external conditions, e.g., trees.

\subsubsection{Facilities architecture}

The school facilities where the studies were conducted consist of three two-storey wings forming a letter $\mathrm{U}$. The two twin wings contain lecture rooms and technical laboratories. The rooms have access to the solar radiation from $\mathrm{S}$ and $\mathrm{N}$. The central halls on the first and the second floor do not have access to the daylight. The third wing, joining the twin wings of similar size and layout of rooms, contains a traffic route, an auditory and technical rooms.

All lecture rooms have a layout typical for education facilities, with the external wall consisting of window joinery in ca. $80 \%$. A view of an architecture of the whole School Complex No. 1 in Swarzędz and its orientation in terms of the Sun movement are presented in Figure 1. Currently, a construction of a new educational part that will form an integral part of the whole facilities is being completed. It is outlined in Figure 1 with a dashed line.

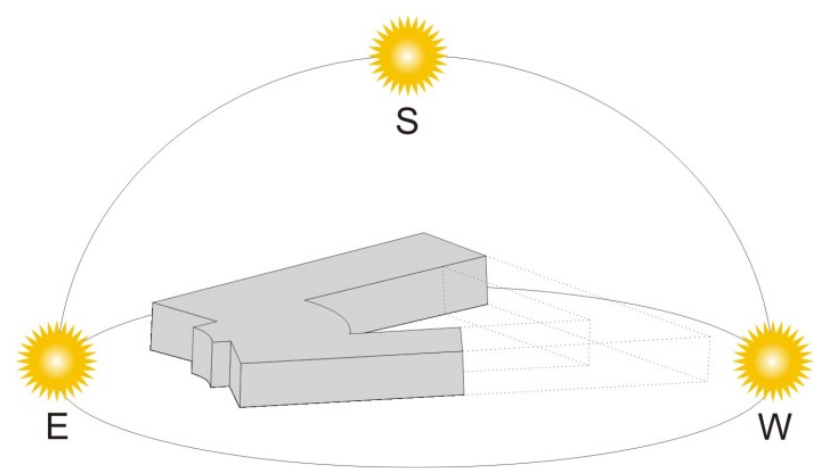

Fig. 1. An illustrative architecture of the ZS1 object in Swarzędz with the apparent solar movement shown.

\subsection{Photovoltaic system}

The photovoltaic system was installed on a roof of the middle part of the school facilities. It consists of 4 polycrystalline panels, of $250 \mathrm{~W}$ each. A construction, on which all PV panels were installed, was prepared for installation of a tracking system. Solar radiation intensity sensors send a message to a control device. Following a data analysis, the controller send information to a system controlling motors of the horizontal and the vertical axes to direct the set of panels towards the largest intensity of the solar radiation. Another device included in the photovoltaic system is the inverter. At this stage, a charging controller and a battery pack were not installed. A construction of power storage capacities is foreseen in the next stage of the solar system expansion. The system operates in the off-grid system, meaning, the generated electric power is not supplied to an external grid. The energy demand of the whole facility is so large that even at a peak of the power generation, during which the panels can produce the power of $1 \mathrm{~kW}$, the whole electric power is used by devices operating at the facilities. Figure 2 shows an example of a diagram of the installed solar system. The dashed lines show components that will be connected and started in the future, that is, the tracker controller, the charging regulator, and batteries.

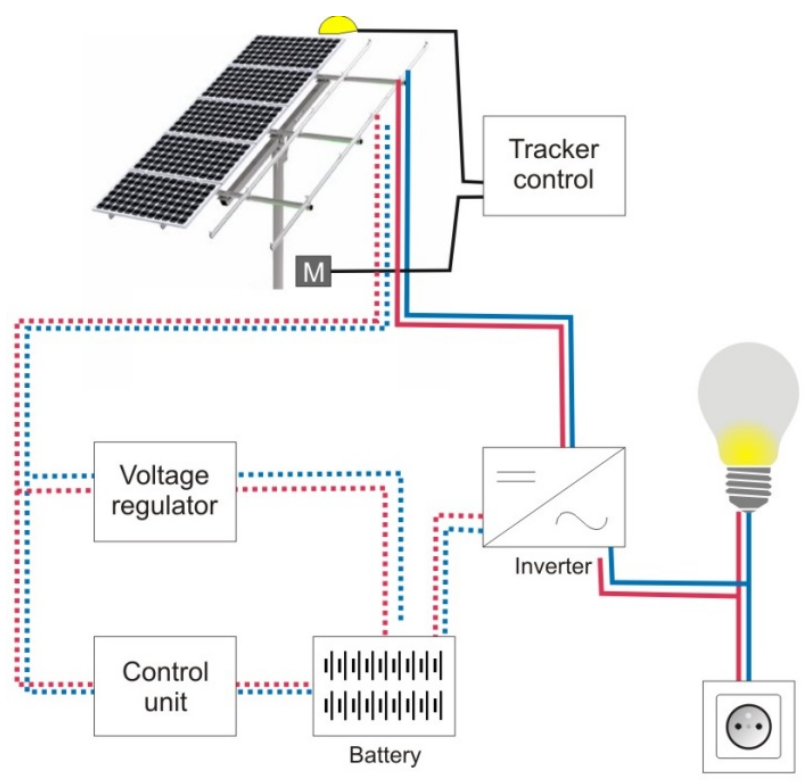

Fig. 2. PV system diagram.

\section{Measurements of light intensity in rooms}

Measurements were conducted in rooms located on $\mathrm{S}$ and $\mathrm{N}$ sides. The rooms are located on a second floor and are the same in terms of window and floor surface areas. The light intensity data was recorded in different weather conditions, for 18 months. This way an extensive range of data was obtained which was used to determine characteristics of the light intensity distribution in rooms. Simultaneous measurements conducted in rooms located on opposite sides allowed to compare intensity distributions. The obtained results are presented and discussed in section 3.2 of this paper. 


\subsection{Measurement method and location}

Light intensity data was recorded at 16 points in two rows, at $1 \mathrm{~m}$ intervals, separately for each room. This measurement method was selected to eliminate possible measurement errors. The measurements were conducted manually, using a professional light meter. A disadvantage of this method is low measurement precision resulting from a need to precisely operate the light sensor, and a long time of measurement. Currently, a measurement matrix was developed and works on starting the whole measurement system are conducted. The measurement matrix will consist of light intensity sensors installed on 16 stable measurement supports. The light intensity values will be recorded simultaneously for all 16 sensors. This way errors occurring mainly during measurements of light intensity on very cloudy days will be eliminated.

\subsection{Measurement results}

The conducted measurements of light intensity distributions show similar courses of characteristics for rooms located on $\mathrm{N}$ and on $\mathrm{S}$. This means that the light intensity distribution in a room does not depend on the building orientation. The obtained results can be used to draw conclusions concerning the intensity of external light ensuring the required lighting intensity inside, in a given place in the room. This data is useful in development and use of appropriate algorithms for control of lighting for ex. in smart buildings [2]. Figure 3 shows examples of the light intensity distribution in the room on the $\mathrm{S}$ side. The characteristics show light intensity distributions in various weather conditions., The measurements were carried out in March 2017. Analogous data for the room on the $\mathrm{N}$ side is shown in Figure 4. On the charts showing the illumination in the rooms from the south and north sides, the values for illumination demanded by law were marked. For such rooms it is 500 lux. By comparing a course of characteristics in both charts it can be said that the room orientation does not significantly influence the light intensity distribution in a room. The charts show that in worse weather conditions the course of the light intensity distribution is similar as in good weather conditions. An obvious difference concerns higher variations in the intensity at the initial stage, for the large intensity. Whereas, for example, in a case of the cloudy sky, when the solar radiation intensity is lower, the course of the characteristic course is smoother at the initial phase. The obtained characteristics can also be used to conclude that the change in the light intensity deeper in the room is significantly lower that at the initial distance near the window. Problems with measurements in a case of significant variability in the solar radiation intensity, for example, on cloudy days, should also be mentioned. Differences in the light intensity can reach an amplitude even of several thousand luxes in just a few seconds.

Recordings of the electric power generated in the installed photovoltaic panels showed that the photovoltaic system in the school building generated 937 $\mathrm{kWh}$ of the electric power during 12 months. Of course, starting of the tracker control system will possibly increase the amount of the generated energy.

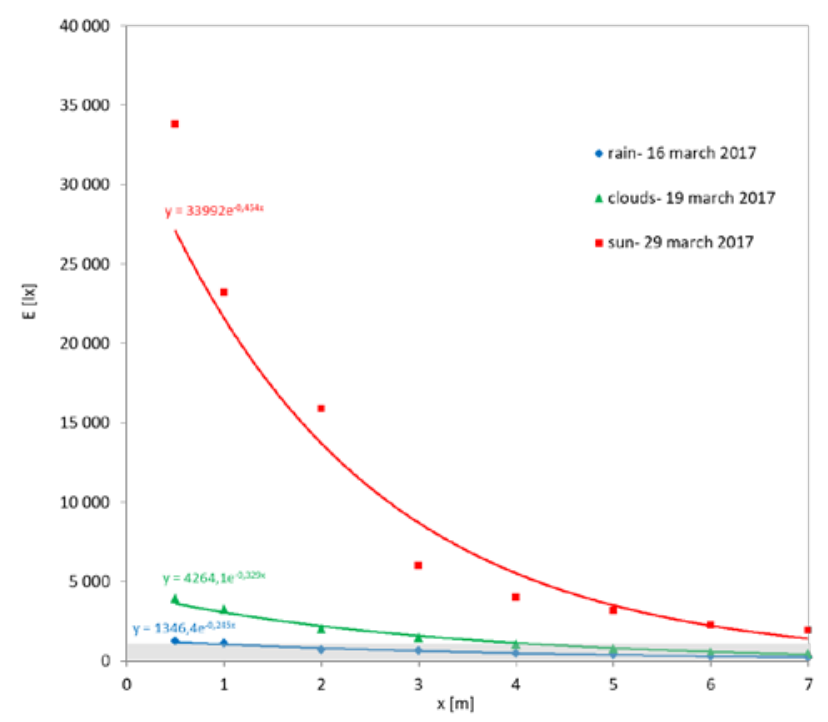

Fig. 3. Distribution of daylight in the room from the South side.

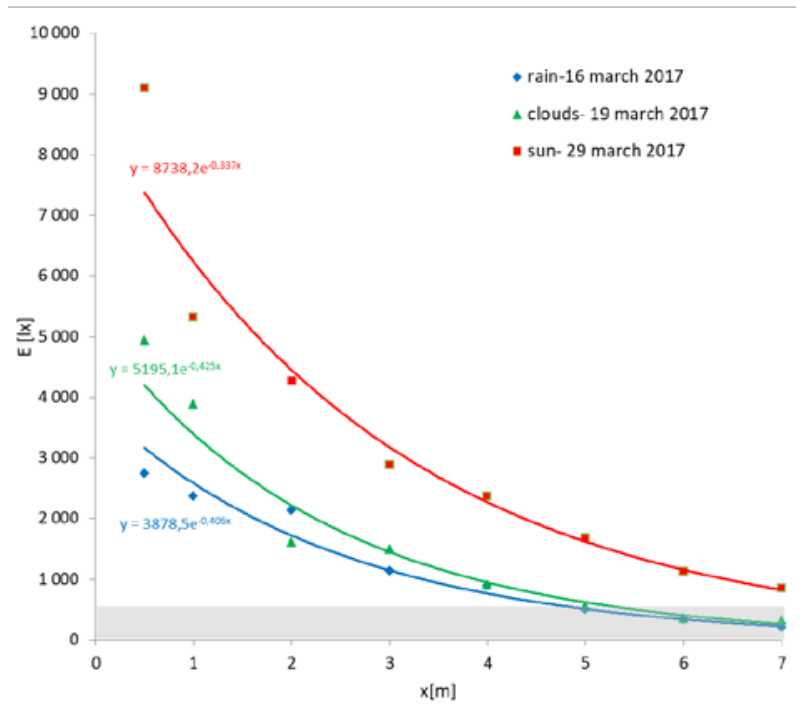

Fig. 4. Distribution of daylight in the room from the North side.

\section{Savings resulting from the use of the solar radiation}

Energy consumption for lighting represents from 10 to $20 \%$ of total energy costs at facilities [3]. Therefore, actions aiming at savings in energy consumption by lighting systems are a very important issue. The conducted studies on the light intensity distribution in rooms allow to estimate savings that can be achieved by using the solar radiation. The obtained results were used to determine the value of the solar radiation intensity at which we can switch artificial lighting off or reduce its power. Limit values for the solar radiation intensity at which the required lighting intensity is ensured at a relevant depth of a room are shown in Table 1 . It 
includes a classification into the light intensity outside, $\mathrm{E}_{\text {out }}$, and inside, $\mathrm{E}_{\mathrm{in}}$. The parameter $\mathrm{x}$ expressed as $[\mathrm{m}]$ means a room depth measured from the window. The obtained results were used to conclude that at the light intensity below $7000 \mathrm{~lx}$ outside or of $1200 \mathrm{~lx}$ inside near a window, the required light intensity is not ensured at the whole depth of the room, that is, it is necessary to switch all installed sources of artificial lighting on. Whereas, when the solar radiation intensity exceeds $12000 \mathrm{~lx}$ outside and $3200 \mathrm{~lx}$ inside near a window, the required light intensity is provided in the whole room, and all installed sources of artificial lighting can be switched off. Other intensity values for which the required lighting intensity is ensured at a relevant depth of a room are provided in Table 1. The lighting control system, which will switch the artificial lighting system on/off and control its power, should be selected according to these criteria.

The obtained measurement results allowed to estimate savings that can be obtained by implementation of an appropriate control system. The conducted calculations show that the use of solar radiation for lighting of rooms can reduce the electric power consumption for lighting by $45-48 \%$, depending on a control system used [3]. Use of appropriate lighting control algorithms is important, as they will ensure an optimal control of selected sources of artificial lighting in a room [4]. The existing control systems in the venues are also used for effective energy consumption in smart buildings [5].

Table 1. Values of the solar radiation intensity ensuring a required light intensity at a specified distance from a window.

\begin{tabular}{|c|c|c|c|c|c|}
\hline \multirow{2}{*}{$\begin{array}{c}\mathrm{X} \\
{[\mathrm{m}]}\end{array}$} & $\begin{array}{l}E_{\text {out }} \\
{[\mathrm{lx}]}\end{array}$ & $<7000$ & $\begin{array}{c}7000- \\
9000\end{array}$ & $\begin{array}{c}9000- \\
1200\end{array}$ & $>12000$ \\
\hline & $\begin{array}{l}\mathrm{E}_{\text {in }} \\
{[\mathrm{lx}]}\end{array}$ & $<1200$ & $\begin{array}{c}1200- \\
2300\end{array}$ & $\begin{array}{c}2300- \\
3200\end{array}$ & $>3200$ \\
\hline 2 & & $\mathrm{ON}$ & OFF & OFF & OFF \\
\hline 4 & & ON & ON & OFF & OFF \\
\hline 6 & & ON & ON & ON & OFF \\
\hline
\end{tabular}

\section{Conclusions}

The solar radiation has a high energy potential that can be used to improve the energy effectiveness of lighting systems by reducing the electric power consumption for lighting purposes. The first method presented in the paper concerns the use of the daylight to light rooms, with simultaneous reduction in artificial lighting [6]. The required light intensity in a room can be ensured by switching relevant selected sources of artificial lighting on. The solar radiation intensity distributions in a room can be useful for development of the lighting control algorithms. The obtained study results allow us to ensure appropriate power control and regulation for individual lighting fixtures. In consequence, we can achieve savings reaching nearly $50 \%$ of the total power consumption for lighting. However, it should be remembered that when no lighting control system is used, the availability of the solar radiation will not influence savings in the electric power consumption. A problem of lighting system control in conditions of significant variability of the radiation intensity still remains to be solved. In such cases, lack of an appropriate control algorithm can result in flickering of artificial lighting, and this is an undesirable phenomenon having adverse effects both on operation of the lighting system, and on people.

The second method for use of the solar radiation energy is its conversion into the electric power in a photovoltaic system. The solar radiation cannot be stored directly. However, we can store the solar energy transformed into the electric power. When a battery pack is used, it can supply the electric power at times when the PV panels do not generate power, e.g., at night. The system developed this way can lead to significant reductions in the electric power consumption for lighting. According to the current Directives [7], this approach is a recommended action for improvement in the energy efficiency of the facilities, and, in consequence, reduces consumption of the primary energy, and this directly translates to a greater protection of the natural environment.

\section{References}

1. Pr EN:15193 Energy performance of buildings Energy requirements for lighting (2017)

2. M. Kalinowski, T. Zarębski, 2012, Lighting control in smart buildings, Wiadomości elektrotechniczne, 39-42, 10 (2012)

3. S. Sowa, A. Kamińska, 2018. Predicted reduction of energy consumption in the school building by lighting control with KNX system. Przegląd Elektrotechniczny, 193 - 197, 2 (2018)

4. S. Sowa, J. Gielniak, Lighting control algorithms in a school venue using KNX system. Electrical Engineering, 153-162, 96 (2018)

5. M. Horyński, 2013, The application of dispersed processing networks in order to optimize the energy consumption in contemporary buildings, Przegląd elektrotechniczny, 293-295, 4 (2013)

6. E. Plikunas, M. Ziajkowski, Use of solar radiation in shaping the energy efficiency in public buildings, Electrical Engineering, 87 (2016)

7. Directive 2012/27/EU of the European Parliament and of the Council of 25 October 2012 on energy efficiency 2012/27/UE, Brussels (2012) 